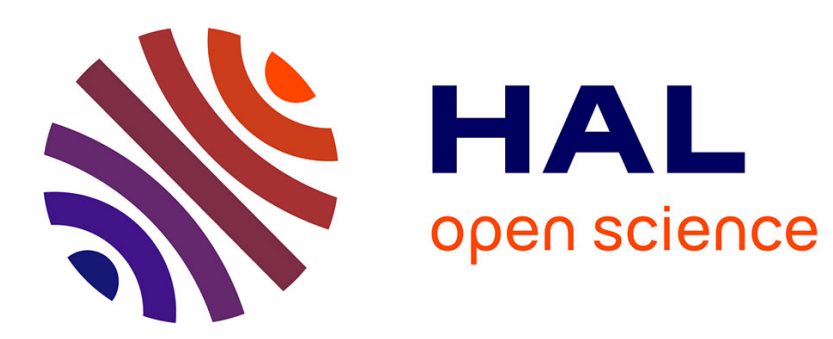

\title{
Commande numérique d'un champ d'héliostats d'une centrale solaire à tour
}

\author{
A. Achaibou, C. Bourdeau
}

\section{To cite this version:}

A. Achaibou, C. Bourdeau. Commande numérique d'un champ d'héliostats d'une centrale solaire à tour. Revue de Physique Appliquée, 1980, 15 (3), pp.383-391. 10.1051/rphysap:01980001503038300 . jpa-00244741

\section{HAL Id: jpa-00244741 https://hal.science/jpa-00244741}

Submitted on 1 Jan 1980

HAL is a multi-disciplinary open access archive for the deposit and dissemination of scientific research documents, whether they are published or not. The documents may come from teaching and research institutions in France or abroad, or from public or private research centers.
L'archive ouverte pluridisciplinaire HAL, est destinée au dépôt et à la diffusion de documents scientifiques de niveau recherche, publiés ou non, émanant des établissements d'enseignement et de recherche français ou étrangers, des laboratoires publics ou privés. 


\title{
Commande numérique d'un champ d'héliostats d'une centrale solaire à tour
}

\author{
A. Achaibou et C. Bourdeau \\ Laboratoire d'Automatique et d'Analyse des Systèmes du C.N.R.S., \\ 7, avenue du Colonel-Roche, 31400 Toulouse, France
}

(Reçu le 31 juillet 1979, révisé le 18 décembre 1979, accepté le 18 décembre 1979)

\begin{abstract}
Résumé. - La régulation du flux de nature variable renvoyé par le champ d'héliostats impose un système de commande assez souple et précis pour minimiser les pertes dues aux erreurs de pointage et pour pouvoir à tout moment agir sur les diverses zones du champ d'héliostats. Par ailleurs, le système doit fonctionner dans les meilleures conditions de sécurité.

Le laboratoire a développé un système de commande numérique de type décentralisé utilisant une structure micro-informatique répartie. Cette structure est une commande décentralisée, hiérarchisée à deux niveaux. Le premier niveau est constitué par des microcalculateurs de zone qui assurent la gestion de zones comprenant vingt héliostats. Le second niveau est constitué par les microcalculateurs d'héliostat, un par héliostat.

De plus le système teste les transmissions d'informations et les microcalculateurs de zone pour détecter les défaillances éventuelles.
\end{abstract}

\begin{abstract}
In a solar central receiver power plant, the regulation of the global flux reflected by the field of heliostats requires an adaptable and precise control system to minimize the losses (partly caused by tracking incertainties), and to control the thermal power collected by the receiver. This system should also yield the best safety conditions.

The laboratory has developed a decentralised numerical control system using distributed microprocessors. This system has two level hierarchical control structure. The first level is defined by the zone-microprocessors, each of them managing about twenty heliostats. The second level consists of the heliostat-microprocessors.

All the zone-microprocessors are connected to a central computer which receives information from them and send them the value of control parameters and instructions on the basis of checking and testing operations.
\end{abstract}

Introduction. - La production d'électricité solaire par la voie thermodynamique sous de fortes puissances ( 3 à $10 \mathrm{MWe}$ ) impose l'utilisation d'un système de capteurs susceptible de collecter sur de grandes surfaces le flux solaire de façon à le concentrer par réflexion dans le volume réduit d'une chaudière.

La centrale à tour permet d'atteindre de telles puissances, elle se compose d'un champ d'héliostats focalisants (300 à 2000 soleils) individuellement asservis, réfléchissant le flux solaire sur une chaudière fixe située au sommet d'une tour (Fig. 1).

Cet article présente une structure de commande du champ de miroirs par micro-informatique répartie, associée à une commande numérique par coordonnées calculées de la position de chaque réflecteur.

L'accent est mis plus particulièrement sur la sécurité de fonctionnement et sur la précision de pointage qui constituent les contraintes essentielles liées à la commande d'un tel système.

L'étude présentée dans cet article s'inscrit dans le cadre du Programme Interdisciplinaire de Recherche

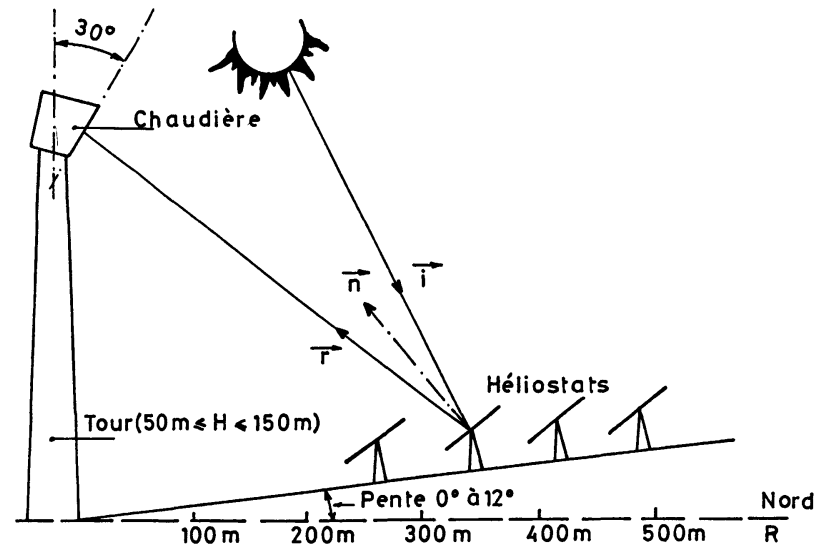

Fig. 1. - Champ d'héliostats et tour.

[Field of heliostats and tower.]

et de Développement de l'Energie Solaire (PIRDES) comprenant la réalisation de la centrale à tour THEMIS, assurée conjointement par le CNRS et l'EDF. 
1. Phénomènes physiques déterminant les conditions de fonctionnement. - 1.1 . CONTRAINTES THERMIQUES ET DE SÉCURITÉ $[1,3]$. — La captation du flux solaire concentré et fluctuant en fonction de l'opacité du ciel nécessitait une modulation de ce flux lors de certaines perturbations; en effet, les actionneurs de la chaudière de par leur positionnement et leur mode d'action sont inaptes à contrecarrer avec un temps de réponse suffisant les dépassements locaux de contraintes (corrosion du fluide caloporteur) dans le cas de variations brusques d'ensoleillement.

La commande du flux dans l'environnement du récepteur est alors principalement guidée par des consignes respectant les contraintes chaudière réparties sur toute la surface des parois du récepteur et les consignes de sécurité. Le flux renvoyé par l'ensemble des héliostats est très important à l'entrée de la chaudière (concentration optique de 1 à 2 500). La forme et les dimensions de la chaudière, qui est à cavité dans le cas de THEMIS, sont déterminées de façon à minimiser les pertes thermiques, d'où la nécessité d'optimiser les dimensions de la tache à l'entrée de la chaudière (réduction des pertes par réflexion et par convection). Les pertes de flux incident en dehors de l'entrée de la chaudière peuvent être réduites en améliorant la focalisation des héliostats et la précision de pointage.

Pour des raisons technico-économiques, on ne peut attribuer une focale particulière à chaque héliostat. Les études menées au LAAS ont montré que le rendement était peu affecté par un découpage du champ correspondant à un nombre réduit de gammes de focales. En particulier, trois focales suffisent à caractériser un champ de $500 \mathrm{~m}$ de profondeur. L'influence de la focale en fonction de la position de l'héliostat dans le champ, du nombre de facettes constituant le réflecteur et de la position du soleil, est analysée en déterminant les points de densité de puissance maximale sur le plan de la chaudière. Dans le cas de THEMIS, la chaudière est constituée d'un parallélépipède rectangle de $4 \times 4 \times 3 \mathrm{~m}$. Une des faces de $4 \times 4 \mathrm{~m}$ est ouverte et constitue l'ouverture de la chaudière. Un anneau de diamètre extérieur $6,5 \mathrm{~m}$ et de diamètre intérieur $4 \mathrm{~m}$, entoure la fenêtre dans son plan. L'ensemble est incliné sur la verticale d'un angle de $35^{\circ}$.

Le rendement de pénétration, rapport entre la puissance incidente dans la chaudière et la puissance incidente sur le plan de la chaudière, est maximal quand la distance focale $f$ se situe dans un voisinage de la distance héliostat-chaudière $d$, et chute rapidement si $f$ est inférieure à $d$.

Quel que soit le critère de sévérité choisi sur la détermination des distances focales, le dépointage intervient ensuite d'une manière sensible dès qu'il dépasse $2 \times 10^{-3}$ rad sur la normale au réflecteur.

Pour un champ d'héliostats focalisants, dont les focales correspondent à quatre gammes $\left(f_{1}=230 \mathrm{~m}\right.$, $f_{2}=390 \mathrm{~m}, f_{3}=470 \mathrm{~m}$ et $f_{4}=530 \mathrm{~m}$ ), la figure 2 illustre l'influence du dépointage sur le rendement

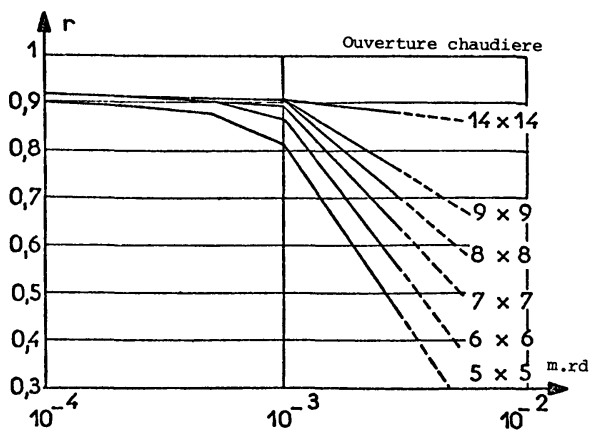

Fig. 2. - Puissance collectée sur un récepteur de $7 \times 7 \mathrm{~m}^{2}=$ $48,700 \mathrm{~kW}$. Ecart-type de dépointage : $1 \mathrm{mrd}$ sur la normale.

[Flux collected by a $7 \times 7 \mathrm{~m}^{2}$ receiver $=48.700 \mathrm{~kW}$. Standard deviation : $1 \mathrm{mrd}$ for the normal.]

global pour différentes ouvertures de chaudière. La figure 3 donne la répartition énergétique dans le plan focal, pour un écart-type de dépointage de $1 \mathrm{mrd}$ sur la normale au réflecteur d'un champ de 1113 héliostats.

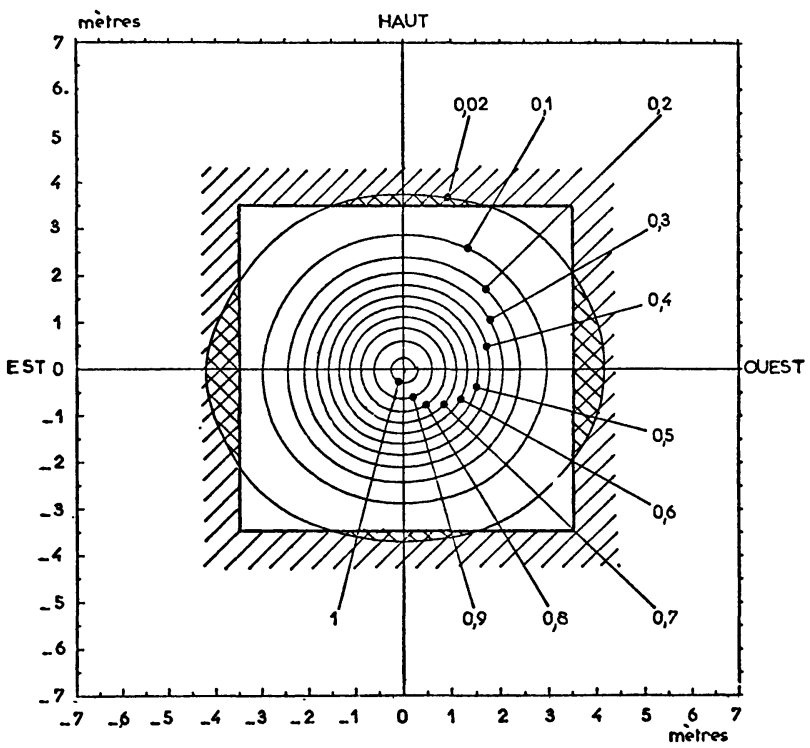

Fig. 3. - Héliostats focalisants.

[Focusing heliostats.]

En concevant la commande du champ d'héliostats à partir d'une décomposition en zones, nous avons pu affecter à chacune un domaine spatial limité de la chaudière; dans ce contexte, il est possible d'élaborer une procédure de commande optimale maximisant l'énergie reçue par la chaudière et opérant le dépointage des zones concernées par les fluctuations du flux solaire incident (Fig. 4).

Les travaux menés au laboratoire sur la modélisation de la commande de flux, ont montré que les temps de réponse sur la modulation du flux solaire ne doivent 


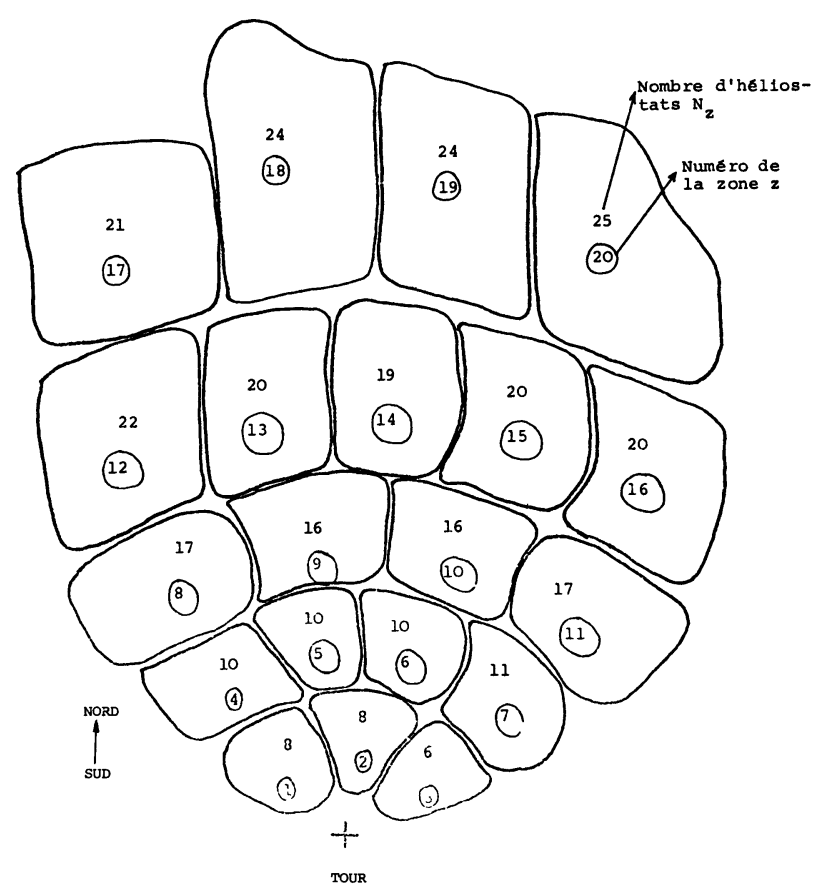

Fig. 4. - Champ du projet THEM1 sur le site de Targasonne, division en zones.

[Field of heliostats for the project THEM1 in Targasonne : zone division.]

pas excéder $3 \mathrm{~s}$ [1]. Par ailleurs, le flux solaire réfléchi par les héliostats constitue aussi un danger pour l'environnement de la centrale et il convient de protéger les structures du haut de la tour et de maîtriser les zones de focalisation lors des trajectoires de démarrages/arrêts pour éviter de créer dans l'espace aérien ou au sol des zones dangereuses non contrôlées.

Dans ces conditions, il est nécessaire que d'une part, l'espace balayé par le flux corresponde à des trajectoires définies en fonction des états du système et que, d'autre part, la formation et la dilution de la tache sur le récepteur soient effectuées successivement pour diverses zones du champ.

On définit aussi une ou plusieurs positions de survie assurant la protection des héliostats contre les agressions externes (vent-grêle) et respectant les contraintes précédentes.

Définition des états du champ. - Le champ est découpé en groupes de 30 héliostats consécutifs affectés à un domaine spatial de la chaudière.

Les états des groupes sont définis en fonction des contraintes précédentes :

TN : travail normal : tous les groupes sont en travail normal : la consigne du point-cible est fixée au foyer $F$ du récepteur solaire. ( $F$ centre géométrique de l'ouverture de la cavité.)

TV : travail variable : tous les groupes sont en travail. La consigne est au point $F+\Delta F(\Delta F$ désignant le dépointage).
TP : travail partiel : certains groupes sont en travail, d'autres en attente.

AN : attente normale : tous les groupes sont en attente.

$\mathrm{AD}:$ attente après déclenchement : tous les héliostats sont en attente après déclenchement du récepteur solaire. (En cas de défaut sur le récepteur solaire, une procédure de déclenchement est initiée par un processeur spécialisé. Tous les héliostats doivent démarrer pour éteindre le flux le plus rapidement possible dans la cavité, et ceci en respectant des trajectoires de sécurité pour la sortie de la tache.)

DS : démarrage en sécurité : les trajectoires des groupes sont définies en tenant compte de leur position de départ.

AS : arrêt en sécurité : tous les groupes en automatique sont immobilisés dans un état tel que la sécurité de l'environnement est assurée.

AM : arrêt maintenance : certains groupes sont en position de lavage. Les autres sont à l'arrêt en sécurité.

SN : sécurité normale : tous les héliostats sont en survie.

SP : survie partielle. Certains groupes sont en survie. Cet état est le résultat du déclenchement d'un groupe ou du déclenchement successif de plusieurs groupes.

Par ailleurs, l'enchaînement des actions sur les groupes est décrit par le graphe suivant (Fig. 5).

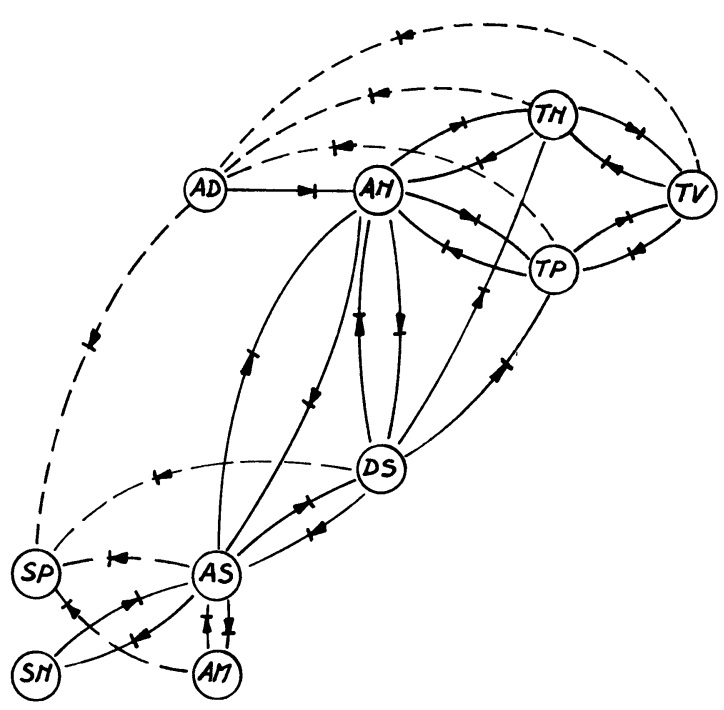

Fig. 5. - Analyse de la logique du champ.

[Analysis of field logics.]

La figure 6 illustre le fonctionnement général; en particulier, les trajectoires de montée et de descente sont implantées sur la paroi nord de l'aérocondenseur.

Les contraintes temporelles essentielles concernent les trajectoires de sécurité au voisinage du récepteur 


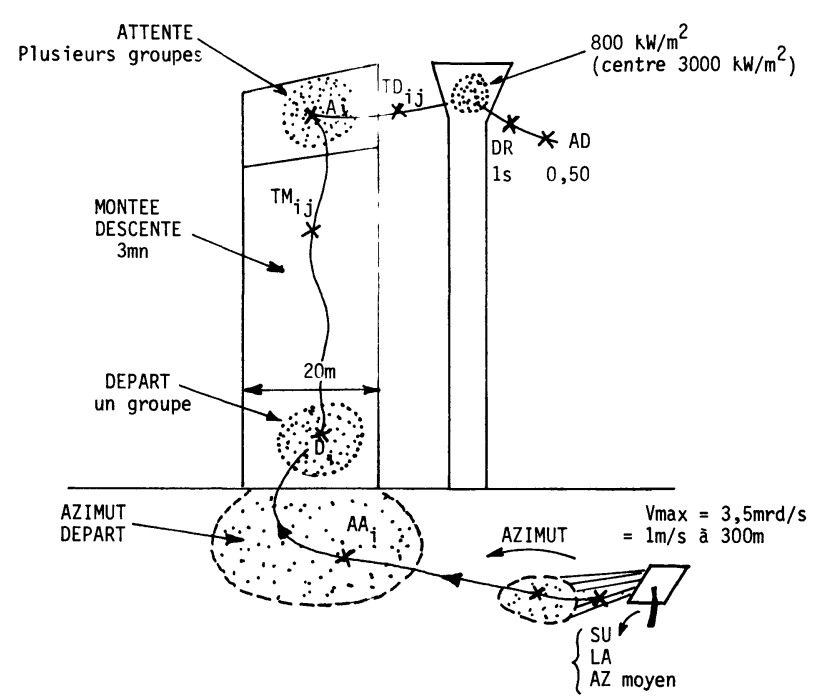

Fig. 6. - Cible, trajectoires.

[Target, trajectories.]

solaire; la zone périphérique de ce dernier ne tolère qu'un flux de $800 \mathrm{~kW} / \mathrm{m}^{2}$, alors que le centre de la tache est à $3000 \mathrm{~kW} / \mathrm{m}^{2}$.

On définit donc des trajectoires de sécurité qui consistent à étaler le flux lors des transitoires correspondant au passage dans cette zone.

Passage normal travail $\rightleftarrows$ attente. - En cas de passage nuageux de courte durée (de l'ordre de la minute) ou de durée moyenne (quelques minutes), le champ doit être partiellement ou totalement dépointé. En effet, dans le premier cas il convient de limiter le choc thermique de réapparition du soleil sur le récepteur en cours d'évolution, et dans le second cas il faut éviter d'envoyer du flux sur la porte du récepteur qui aura été fermée pour limiter la déperdition de chaleur.

Dans ce cas, les groupes rallient des points cibles situés dans la zone d'attente définie sur la figure 6 . Ces commandes doivent être indépendantes pour chaque groupe et exécutées en un temps n'excédant pas $3 \mathrm{~s}$. Les trajectoires correspondantes sont définies par 10 points cibles intermédiaires.

Déclenchement du récepteur solaire. - En cas de défaut sur le récepteur solaire, l'automate de sécurité initie la procédure de déclenchement. Celle-ci est basée uniquement sur la manœuvre du champ; tous les héliostats doivent démarrer pour éteindre le flux le plus rapidement possible dans la cavité en suivant des trajectoires de sécurité définies par deux points situés en avant du récepteur. Le temps mort entre l'envoi de l'ordre et le début de rotation des héliostats doit être inférieur à $1 \mathrm{~s}$.

1.2 Contraintes Relatives a LA PRÉCISION DE DÉPOINTAGE. - Un rendement acceptable, tenant compte des contraintes économiques liées au coût de l'héliostat, nécessite une précision de pointage sur le rayon réfléchi inférieure à $4 \mathrm{mrds}$ qui impose avant tout une période d'échantillonnage voisine de $5 \mathrm{~s}$.
1.3 CONTRAINTES DE SÛRETÉ DE FONCTIONNEMENT. - Compte tenu du danger présenté par le flux concentré pour la chaudière et pour l'environnement de la centrale, tout fonctionnement défectueux sur un héliostat doit pouvoir être détecté de la salle de commande dans un délai inférieur à $6 \mathrm{~s}$.

2. Structures de commande du champ d'héliostats. - Compte tenu des contraintes définies au chapitre précédent, nous avons retenu une structure de commande décentralisée hiérarchisée à trois niveaux. Le champ d'héliostats a été découpé en groupes de 30 héliostats voisins.

Le premier niveau correspond à l'ordinateur central, le deuxième niveau au calculateur de gestion d'un groupe et le troisième niveau au calculateur assurant la commande de l'héliostat (Fig. 7).

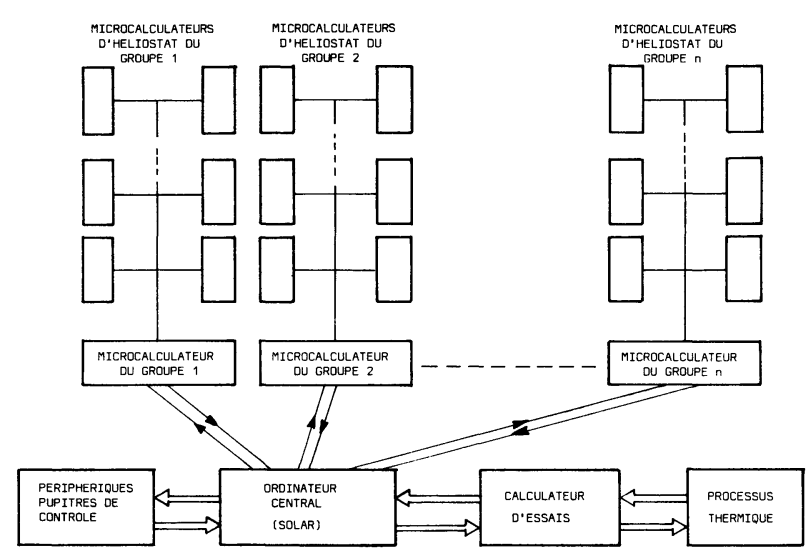

Fig. 7. - Structure du réseau de commande du champ d'héliostats.

[Structure of control network of field of heliostats.]

2.1 FonCtions DE L'ORDINATEUR CENTRAL. L'ordinateur central gère le fonctionnement du champ d'héliostats en élaborant des commandes générales interprétées et exécutées par les calculateurs de groupe.

a) Commandes de contrôle des liaisons : Trois commandes sont affectées au contrôle des liaisons entre calculateurs de groupe et les calculateurs d'héliostats : établissement de la liaison, sa déconnexion, son test.

b) Commandes d'initialisation des calculateurs d'héliostats : Quatre commandes demandent au microcalculateur $(\mu \mathrm{c})$ de groupe le rangement en mémoire vive dans le $\mu \mathrm{c}$ d'héliostat, des paramètres nécessaires aux calculs du temps, de la position du réflecteur et des 16 trajectoires à suivre.

c) Commandes de suivi des trajectoires : Deux commandes : suivi d'une trajectoire définie par initialisation et suivi de la trajectoire de déclenchement $\mathrm{du}$ récepteur solaire.

d) Commandes de lecture et d'écriture de registre d'un calculateur d'héliostat. 
e) Commandes de test : Demandent l'exécution d'un des 16 tests une fois ou cycliquement sur chaque $\mu \mathrm{c}$ d'héliostat.

2. 2 Fonctions DU CALCULATEUR DE GROUPE. Chaque calculateur de groupe ( $\mu \mathrm{c}$ spécialisé) gère le fonctionnement d'un groupe de 30 héliostats. Il interprète des commandes générales provenant de l'ordinateur central et élabore des commandes élémentaires destinées aux $\mu \mathrm{c}$ d'héliostat.

Ces commandes élémentaires définissent une procédure du dialogue inspirée de la procédure SDLC. Elles peuvent être destinées à un ou plusieurs héliostats définis par leur adresse ou bien communes à tous les héliostats du groupe.

a) Commandes de contrôle des liaisons : Trois commandes sont affectées au contrôle des liaisons entre le calculateur de groupe et les calculateurs d'héliostat : établissement, de connexion, test.

b) Commandes d'écriture et de lecture.

c) Commandes de suivi de trajectoire : Déclenchent le suivi de la trajectoire préalablement rangée en mémoire vive dans le $\mu \mathrm{c}$ d'héliostat et provoquent le suivi de la trajectoire de déclenchement du récepteur solaire.

d) Commandes d'acquittement : Indiquent si l'information est bien ou mal reçue. Toutes les commandes précédentes sont suivies d'un acquittement.

e) Commande non acquittée : Elle permet d'écrire des informations directement en mémoire du calculateur d'héliostat. Elle n'est pas suivie d'acquittement.

2.3 Fonctions du CAlCUlateur D'HÉliostat. Le calculateur d'héliostat ( $\mu \mathrm{c}$ spécialisé) interprète et exécute les ordres de commande provenant du calculateur de groupe, calcule la position de la normale au réflecteur et gère l'asservissement des moteurs de position de l'héliostat.

a) Le programme moniteur : Il décode et exécute les commandes et les acquitte si nécessaire.

b) Le programme d'asservissement : L'asservissement est en boucle ouverte par coordonnées calculées de la position du soleil, puis de la position de la normale à chaque héliostat. La position de la normale est définie par les coordonnées du point cible qui peut être modifié sur ordre du calculateur central. Par ailleurs, le programme assure la commande échantillonnée des moteurs de site et d'azimut.

2.4 DéFInItION DES LIAISONS. - Les échanges d'information se font en mode série asynchrone avec synchronisation bit et code détecteur d'erreur; le début et la fin du message sont définis par un drapeau. Ces fonctions sont réalisées par un coupleur universel de transmission (SDLC, HDLC).

- Les liaisons entre les $\mu \mathrm{c}$ de groupe et d'héliostat sont constituées par un bus fonctionnant en mode half-duplex; les échanges sont sous le contrôle du $\mu \mathrm{c}$ de groupe qui a le statut de maître.
- Les liaisons entre l'ordinateur central et les $\mu \mathrm{c}$ d'héliostat sont du type réseau étoile avec double bus; le mode de fonctionnement est le full-duplex.

3. Le protocole de dialogue. - Le protocole choisi s'inspire de la procédure SDLC avec trame non numérotée, puisque toutes les informations correspondant à une fonction peuvent être contenues dans une seule trame. Le fonctionnement est du type appel réponse, c'est-à-dire que chaque trame émise est suivie dès réception par un acquittement.

Nous donnons ci-dessous le format des trames. Une commande comportera donc au moins six octets.

\begin{tabular}{|l|l|l|l|l|l|l|}
\hline 01111110 & & & $\left({ }_{0}\right.$ & & & 01111110 \\
\hline
\end{tabular}
drapeau adresse contrôle informations détection d'erreur drapeau

4. Le logiciel du microcalculateur de groupe. L'exécution de la commande consiste à émettre vers chaque $\mu c$ d'héliostat, dont l'adresse a été spécifiée par l'ordinateur central, des trames de commande relatives à la procédure définie précédemment.

Lorsque tous les $\mu \mathrm{c}$ d'héliostat spécifiés ont été commandés, le $\mu \mathrm{c}$ de groupe émet vers l'ordinateur central une trame d'acquittement définissant la manière dont s'est déroulée l'exécution de la commande. La structure du $\mu \mathrm{c}$ de groupe correspond à cette double fonction de dialogue.

\section{Le logiciel du microcalculateur d'héliostat. -} Il se décompose en trois parties :

- le programme moniteur qui interprète, acquitte et exécute les commandes reçues du $\mu \mathrm{c}$ de groupe,

- le programme de pointage sur cible qui calcule la position de la normale au réflecteur et gère l'asservissement de l'orienteur,

- les programmes de calcul flottant.

Ce logiciel est écrit en assembleur et est implanté sur $4 \mathrm{~K}$ octets de mémoire EPROM.

6. Le programme de pointage sur cible. - 6.1 LE PROGRAMME DE CALCUL DE LA POSITION DU SOLEIL.

- Angle du plan du méridien passant par le soleil par rapport au plan du méridien du lieu :

$$
A H v g=15(T+12)-G+E q T ;
$$

$T$ temps en heure,

$G$ longitude en degré,

$A H v g$ s'exprime en degré.

- Equation du temps :

$$
E q T=E q T_{0}+\sum_{i=1}^{5} a_{i} \cos i \theta+\sum_{i=1}^{5} b_{i} \sin i \theta
$$

avec

$$
\theta=K\left(J+\frac{T}{24}\right)
$$


$J$ est le nombre de jours écoulés depuis une date repère. Ce nombre est calculé chaque jour par l'ordinateur central.

- Déclinaison :

$$
D=D_{0}+\sum_{i=1}^{5} c_{i} \cos i \theta+\sum_{i=1}^{5} d_{i} \sin i \theta
$$

La linéarisation de ces formules conduit à des algorithmes plus simples sans entraîner une dégradation prohibitive de la précision :

$$
\left\{\begin{array}{l}
E q T=E q T_{J}+C_{J} \cdot T \\
A H v g=A_{J} \cdot T+A H_{J} \\
D=D_{J}+E_{J} \cdot T .
\end{array}\right.
$$

Les paramètres $E q T_{J}, C_{J}, D_{J}, E_{J}, A H_{J}$ et $A_{J}$ sont déterminés chaque jour par l'ordinateur central :

$$
\left[\begin{array}{l}
E q T_{J}=E q T_{0}+\sum_{i=1}^{5} a_{i} \cos i K J+b_{i} \sin i K J \\
C_{J}=\frac{K}{24} \sum_{i=1}^{5} \cos i K J-a_{i} \sin i K J \\
D_{J}=D_{0}+\sum_{i=1}^{5} c_{i} \cos i K J+d_{i} \sin i K J \\
E_{J}=\frac{K}{24} \sum_{i=1}^{5} d_{i} \cos i K J-c_{i} \sin i K J \\
A H_{J}=180-G+E q T_{J} \\
A_{J}=15+C_{J}, \quad \text { avec } \quad K=\frac{2}{365,25} .
\end{array}\right.
$$

L'ordinateur central calcule $J, E q T_{J}, C_{J}, D_{J}, E_{J}$, $A H_{J}$ et $A_{J}$ à partir des éphémérides.

Le $\mu c$ d'héliostat calcule $A H v g, D$, les angles de site et d'azimut du soleil.

Précision de calcul : Les erreurs maximales sont de $0,25 \mathrm{mrd}$ sur le site et de $0,15 \mathrm{mrd}$ sur l'azimut.

Influence de la réfraction : La réfraction introduit une erreur inférieure à $1 \mathrm{mrd}$ lorsque la hauteur du soleil au-dessus de l'horizon dépasse $20^{\circ}$; on peut alors effectuer une correction linéaire de $0,5 \mathrm{~min}$ d'arc pour $10^{\circ}$.

6.2 Programme de Calcul de la normale aU RÉFLECTEUR. - L'orienteur constitue une monture alt-azimut, les deux grandeurs de commande sont l'angle de site $\theta_{\mathrm{n}}$ et l'angle d'azimut $\varphi_{\mathrm{n}}$ de la normale au réflecteur, $\theta_{\mathrm{n}}$ compté positif de l'horizontale vers la verticale, $\varphi_{\mathrm{n}}$ compté positif du nord au sud dans le sens des aiguilles d'une montre.

La position de la normale dépend de la position de la cible $C\left(x_{\mathrm{c}}, y_{\mathrm{c}}, z_{\mathrm{c}}\right)$ et de la position du centre de l'héliostat $H\left(x_{H}, y_{H}, z_{H}\right)$.

6.3 CORRECTION DES DÉFAUTS MÉCANIQUES DU REPÈRE D'AFFICHAGE. - Le repère d'affichage constitué par les deux codeurs de site et d'azimut $\mathcal{R}_{\mathrm{A}}$ est un repère non orthonormé décalé par rapport au repère de calcul $\Re_{C}$ en raison des défauts de la structure mécanique.

Il en résulte que si l'on affiche dans ce repère les positions de commande $\varphi_{\mathrm{n}}$ et $\theta_{\mathrm{n}}$ calculées dans le repère $\mathcal{R}_{\mathrm{C}}$, la tache se trouve déviée dans des proportions qui dépendent des défauts mécaniques et de la date.

Il est possible de corriger cette déviation par calcul en identifiant par étalonnage les défauts mécaniques de chaque héliostat et en calculant la position de la normale dans le repère d'affichage $\mathcal{R}_{\mathrm{A}}: \varphi_{\mathrm{n}}^{*}$ et $\theta_{\mathrm{n}}^{*}$.

L'étalonnage des héliostats est effectué automatiquement sur une cible passive. Quatre paramètres suffisent à caractériser les défauts des axes de commande : $\left(\varepsilon_{x_{1}}, \varepsilon_{x_{2}}\right)$ pour ceux en site et $\left(\varepsilon_{z_{1}}, \varepsilon_{z_{2}}\right)$ pour ceux d'azimut. La loi de passage du repère de calcul $\mathcal{R}_{\mathrm{C}}$ au repère d'affichage $\mathcal{R}_{\mathrm{A}}$ est complexe mais peut être simplifiée par des développements limités (2), ce qui permet leur implantation sur un microcalculateur.

L'algorithme de correction se traduit par les expressions :

$$
\begin{gathered}
\left(\varphi_{\mathrm{n}}\right)_{\mathcal{R}_{\mathrm{A}}}=\left(\varphi_{\mathrm{n}}\right)_{\mathcal{R}_{\mathrm{C}}}+\Delta \varphi_{\mathrm{n}} \\
\left(\theta_{\mathrm{n}}\right)_{\mathcal{R}_{\mathrm{A}}}=\left(\theta_{\mathrm{n}}\right)_{\mathcal{R}_{\mathrm{C}}}+\Delta \theta_{\mathrm{n}} \\
{\left[\begin{array}{c}
\Delta \varphi_{\mathrm{n}} \\
\Delta \theta_{\mathrm{n}}
\end{array}\right]=\left[\begin{array}{llll}
a_{11} & a_{12} & a_{13} & a_{14} \\
a_{21} & a_{22} & a_{23} & a_{24}
\end{array}\right]\left[\begin{array}{c}
\varepsilon_{x 1} \\
\varepsilon_{x 2} \\
\varepsilon_{z 1} \\
\varepsilon_{z 2}
\end{array}\right]=A(t) . \varepsilon .}
\end{gathered}
$$

Nous ne donnons aucune expression analytique sur la loi de correction, l'ensemble de ces travaux faisant l'objet d'une licence ANVAR. Cette méthode permet de corriger des défauts allant jusqu'à $30 \mathrm{mrd}$.

La validité de l'algorithme a été vérifiée par simulation en comparant d'une part les corrections obtenues à l'aide d'un programme numérique n'effectuant aucune approximation et, d'autre part, celles obtenues à partir de l'algorithme précédent (Fig. 11).

Les coefficients $a_{i j}$ de la matrice sont dynamiques; ils dépendent de la position de l'héliostat sur le site et de celle du soleil.

La validité de cet algorithme a été évaluée par simulation pour différentes configurations de l'héliostat sur le site et par des essais sur un héliostat réel pour différentes dates.

Les défauts de chaque héliostat sont identifiés à partir d'un nombre $n$ de mesures des déviations $\left(\Delta \varphi_{\mathrm{r}}, \Delta \theta_{\mathrm{r}}\right)$ du centre de la tache lumineuse sur une cible passive. La précision de l'identification du vecteur $\varepsilon$ sera d'autant meilleure que $n$ sera grand.

Néanmoins, $n$ doit demeurer relativement faible pour que le temps d'étalonnage n'atteigne pas une valeur prohibitive au niveau de l'ensemble du champ. Aussi avons-nous été amenés à définir une stratégie d'étalonnage pour chaque groupement d'héliostats.

Nous donnons ci-dessous quelques résultats obtenus : 


\section{- Par simulation :}

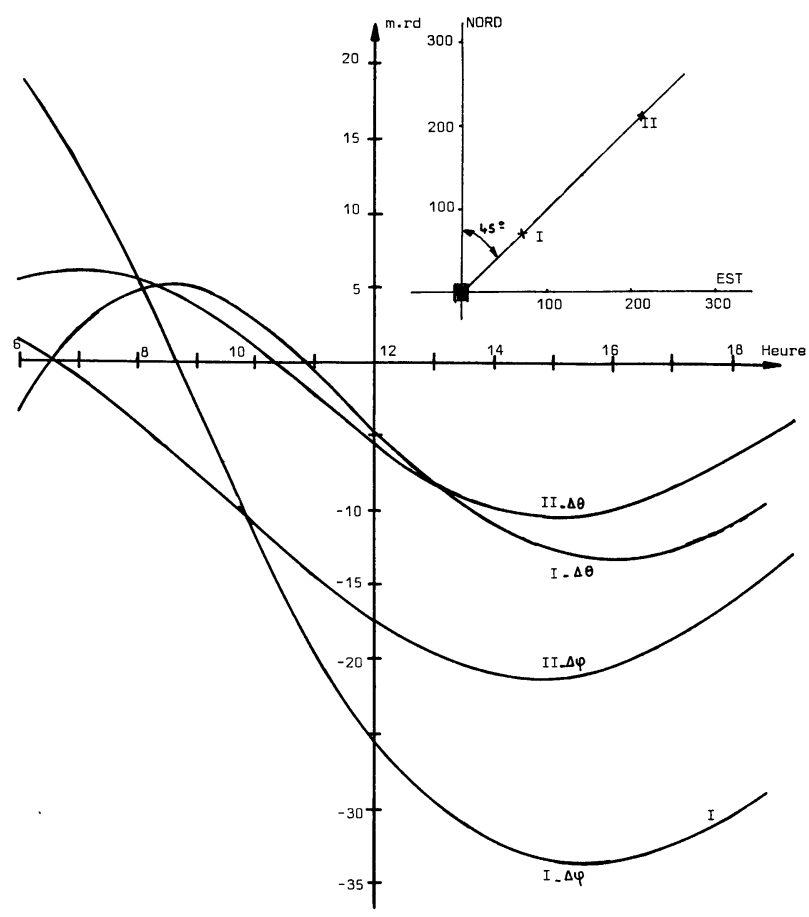

Fig. 8. - Dépointage : $\Delta \theta_{\text {site }}-\Delta \varphi_{\text {azimut }}$.

[Dispointing : $\Delta \theta_{\text {site }}-\Delta \varphi_{\text {azimut }}$.]

La figure 8 donne les déviations en site et en azimut du centre de la tache lumineuse dans le cas d'un héliostat ayant des déformations

$$
\varepsilon_{x_{1}}=\varepsilon_{x_{2}}=\varepsilon_{z_{1}}=\varepsilon_{z_{2}}=10 \mathrm{mrd},
$$

au solstice d'été, occupant les positions I et II.

Les figures 9 et 10 représentent les déviations résiduelles dans le cas où l'identification a été effectuée à partir de 14 et de 5 points de mesure.
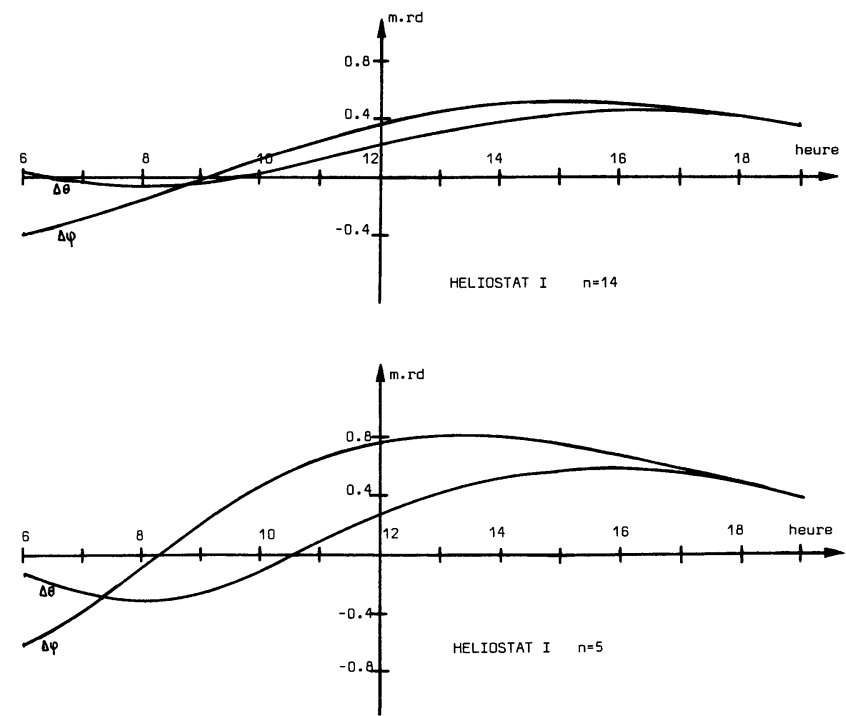

Fig. 9. - Dépointages résiduels. Héliostat I.

[Residual dispointing. Heliostat I.]
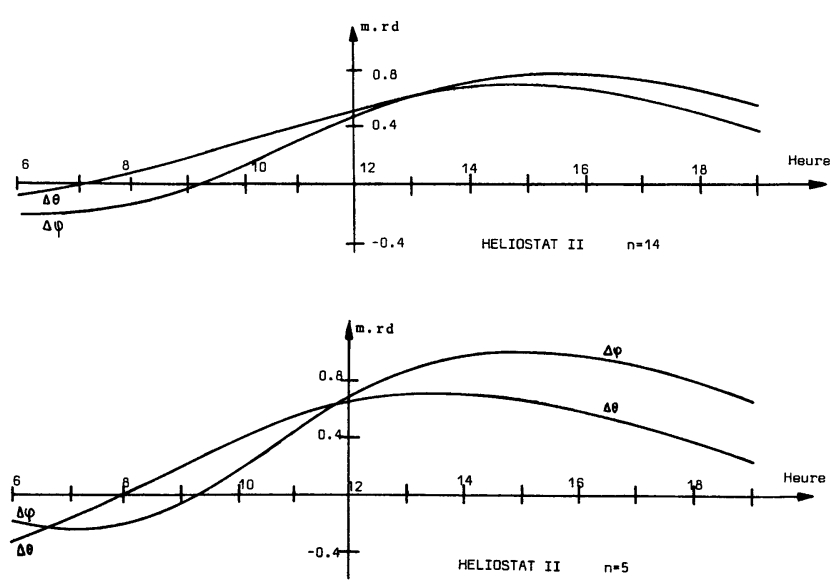

Fig. 10. - Dépointages résiduels. Héliostat II.

[Residual dispointing. Heliostat II.]

Cas réel. - Nous donnons un exemple concret d'étalonnage d'un héliostat à l'échelle 1 , implanté au LAAS. La cible est constituée par un pan de mur équipé de repères quadrillés située à $300 \mathrm{~m}$ de l'héliostat. Par rapport à un repère du type $\mathcal{R}_{0}$ centré sur l'héliostat, l'azimut et le site de la cible sont respectivement de $160^{\circ}$ et de $5^{\circ}$.
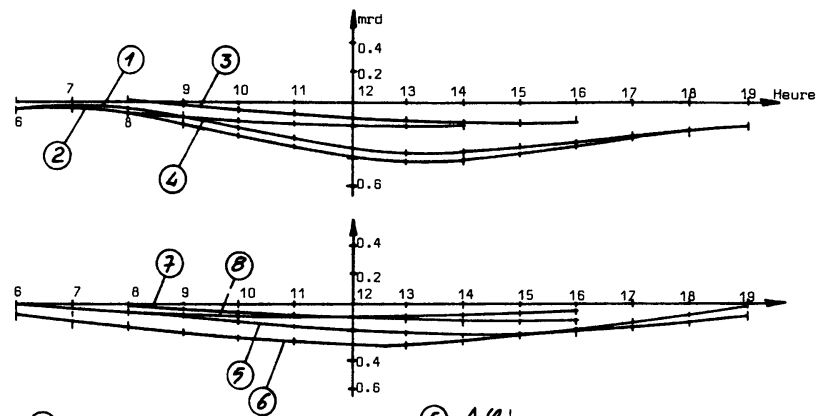
(1) $\Delta \varphi$ Hel1ostat $-100 \mathrm{~m}-45^{\circ}$
(5) $\Delta \varphi$
(2) $\Delta \theta$
(6) $\left.\Delta \theta\right|_{\text {le } 21.06} ^{\text {Helliostat }}$
(3) $\left.\Delta \varphi\right|_{\text {Heliostat }-100 \mathrm{~m}-45^{\circ}}$
(7) $\Delta \varphi$
(4) $\left.\Delta \theta\right|_{1 \mathrm{e} 21.12} ^{\text {Hellostat }}$
(8) $\left.\Delta \theta\right|_{1 \mathrm{e} 21.12} ^{\text {Héliostat }-300 \mathrm{~m}-45^{\circ}}$

Fig. 11. - Dépointage résiduel entre le développement et l'algorithme numérique.

[Residual dispointing between development and numerical algorithm.]

La figure 12 représente les points de mesure lus sur la cible, le 11.09.78. Sur la figure 13 nous avons tracé :

- les courbes de déviations déduites des points de mesure (lissage),

- les écarts corrigés à partir d'une identification des défauts correspondant à 5 points de mesure.

Dans la pratique, l'étalonnage du champ sera réalisé à partir de cinq mesures (deux le matin, une à midi et deux l'après-midi) de déviation de la tache générée par chaque héliostat sur une cible passive. 


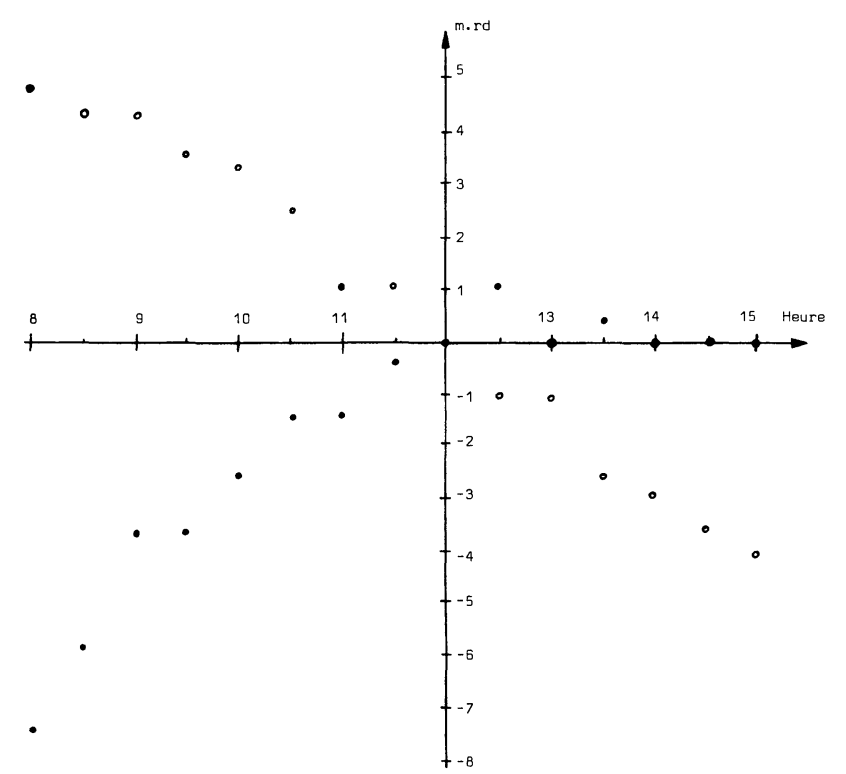

Fig. 12. - Courbe d'étalonnage de la tâche.

[Calibration curve of the spot.]

Les dépointages correspondants seront définis à partir des informations fournies par une caméra de télévision. Les trajectoires d'étalonnage sont séquencées par le système de commande du champ.

6.4 Programme D'ASSERvissement. - L'orienteur est entraîné par deux moteurs continus autour des axes de site et d'azimut.

La position de ces deux axes est repérée par deux codeurs numériques liés au sol et dont les axes sont liés aux axes de l'orienteur.

Le microcalculateur d'héliostat effectue l'acquisition des grandeurs angulaires délivrées par les codeurs,

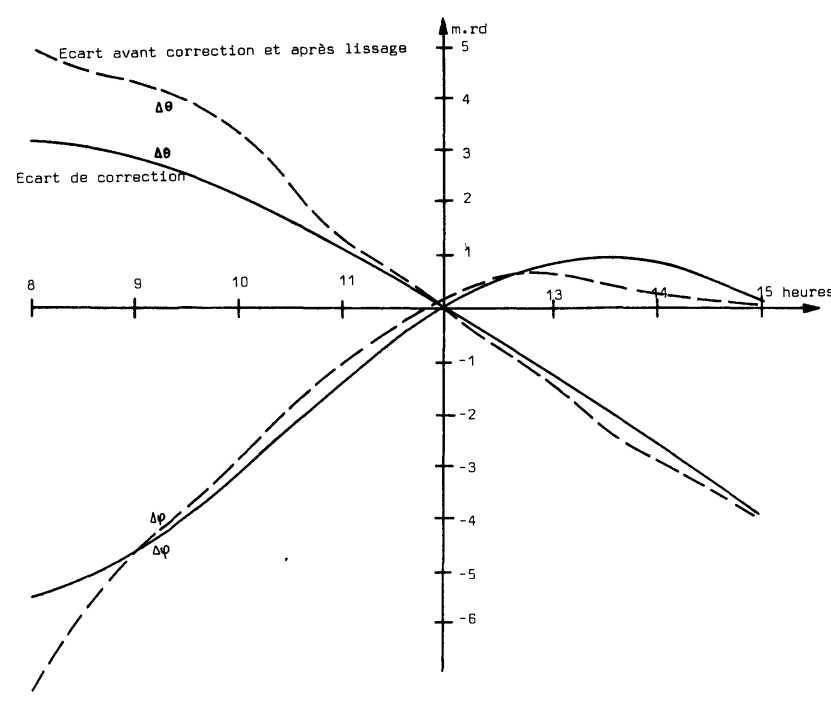

Fig. 13. - Efficacité de la correction dans un cas réel.

[Correction efficiency in real case.] à chaque période d'échantillonnage ; il compare alors les positions lues et les positions calculées.

Lorsque l'écart est en dehors d'un seuil donné, les moteurs sont commandés à pleine puissance.

Lorsque l'écart est compris dans le seuil, les moteurs sont commandés pendant un temps proportionnel à l'écart.

7. Détection des défaillances. - Les défaillances peuvent se produire à six niveaux :

- au niveau de l'ordinateur central,

- au niveau des liaisons entre l'ordinateur central et les microcalculateurs de groupe,

- au niveau des microcalculateurs de groupe,

- au niveau des liaisons entre les microcalculateurs de groupe et les microcalculateurs d'héliostat,

- au niveau des microcalculateurs d'héliostat,

- au niveau de l'asservissement des héliostats.

Certaines de ces défaillances seront détectées en raison de leur incohérence, soit par le code détecteur d'erreur s'il s'agit d'une erreur de transmission, soit par le logiciel s'il s'agit d'un code non reconnu.

Ces défaillances sont donc détectées par la procédure de dialogue choisie et entraînent un rejet.

Par contre, certaines défaillances échappent à ce contrôle et ne peuvent être décelées que par des tests de surveillance effectués aux différents niveaux.

7.1 Défaillances DÉtectées Par la PROCÉDURE DE DIALOGUE. - Ces défaillances concernent d'une part, les erreurs de transmission et, d'autre part, les erreurs de codage qui peuvent se produire à l'intérieur des microcalculateurs.

Erreurs de transmission. - Les erreurs de transmission dans un tel système se traduisent par l'inversion d'un ou plusieurs digits. Ce phénomène est dû aux parasites affectant la ligne de transmission.

La ligne est bifilaire torsadée blindée et constitue un support de transmission binaire efficace et peu coûteux.

Enfin, la transmission sur deux fils et l'utilisation d'émetteurs récepteurs de ligne travaillant en mode différentiel permet de rejeter les parasites dus au mode commun existant entre les masses des circuits d'émission et de réception séparés par des distances importantes.

Par ailleurs, l'utilisation de codes cycliques détecteurs d'erreur accroît la sécurité de la transmission en décelant la plus grande partie des erreurs résiduelles.

Le contrôle d'erreur choisi est celui de la procédure SDLC ; il consiste en l'introduction de deux octets de bits redondants dans les messages à transmettre.

Ce code est cyclique et séparable, il est puissant et bien adapté à la détection d'erreurs indépendantes groupées en paquets (code CRC cyclic redundancy check code).

Le polynôme générateur retenu est :

$$
x^{16}+x^{12}+x^{5}+1 \text {. }
$$


Ces performances sont les suivantes :

- distance de Hamming de 4,

- détection de tout paquet d'erreurs ne dépassant pas 16 bits de longueur,

- détection de nombre impair d'erreurs,

- détection de tout ensemble de deux erreurs.

Le tableau suivant montre l'efficacité de ce code en donnant le taux résiduel d'erreur $(\xi)$ en fonction de la probabilité d'erreur sur un bit $(\mathrm{Pe})$.

$$
\begin{aligned}
& \mathrm{Pe} \\
& \begin{array}{cc}
\text { Pe } & 10^{-4} \\
\bar{\xi} & 6,2 \times 10^{-10}
\end{array} \\
& \frac{10^{-5}}{-} \frac{-}{6 \times 10^{-13}} \\
& 10^{-6} \\
& 6,2 \times 10^{-16}
\end{aligned}
$$

Le traitement du code détecteur d'erreur est effectué par le coupleur SDLC.

Reprise en cas de panne. - Dans tous ces cas, le logiciel de traitement procède au rejet de la trame erronée, d'une part, en renvoyant à la station émettrice une commande de rejet indiquant le type d'erreur et, d'autre part, en ne prenant pas en compte cette information.

Après réception du rejet, la station émettrice émet une nouvelle fois la trame.

7.2 DéFaILlances détectées a PARTIR DE TEST DE SURVEILLANCE. - Afin d'accroître la sécurité du système de commande, il est nécessaire d'appliquer une stratégie de tests périodiques capables de déceler un fonctionnement anormal en moins de $6 \mathrm{~s}$.

La notion de test d'un élément du système recouvre en fait deux problèmes de niveaux différents :

1) le problème le plus important concerne l'observabilité d'une panne;

2) le deuxième problème concerne la localisation de ce défaut.

Le premier problème sera traité de manière automatique en raison des contraintes de temps liées à la montée en température de la chaudière, le second est sous le contrôle de l'opérateur, il est traité lorsque le système ne présente plus de danger. Le logiciel correspondant constitue une aide à la maintenance, il consiste à déclencher une suite de tests par des fonctions choisies par l'opérateur.

La détection des pannes se fait au moyen de tests déterminés, appliqués cycliquement dans un ordre de complexité croissante. Ces tests permettent de s'assurer $\mathrm{du}$ bon fonctionnement des divers niveaux de la commande.

Ils sont générés sur ordre de l'ordinateur central.

Surveillance des héliostats. - Deux types de tests sont effectués permettant de déceler :

- une panne d'asservissement (moteurs, codeurs, ...),

- une panne du microcalculateur d'héliostat.

Surveillance des microcalculateurs de groupe. - Le bon fonctionnement du microcalculateur de groupe est vérifié par une suite de tests effectués cycliquement aux divers niveaux du logiciel et du matériel.

Ces tests sont déclenchés par l'ordinateur central.

La détection d'un défaut entraîne une mise en position de sécurité automatique et immédiate selon la stratégie définie dans le cas de pannes décelées par la procédure.

8. Conclusion. - La structure de commande décrite dans ce document permet de résoudre les problèmes d'automatisation d'un champ d'héliostats avec une sécurité acceptable compte tenu des contraintes financières (la commande ramenée au niveau de chaque héliostat doit coûter moins de $20000 \mathrm{~F}$ ).

La structure de commande décentralisée possède par rapport à une structure centralisée les avantages suivants :

- souplesse d'utilisation dans la réalisation de fonctions complexes telles que suivi de trajectoires, exécution de test ou régulation du flux lumineux,

- possibilité de surveillance du champ à partir du même réseau de commande, donc sans augmentation de coût,

- diminution des contraintes temporelles en décentralisant et en hiérarchisant les taches; le temps libre est mis à profit pour accroître la surveillance du champ,

- amélioration de la sécurité et de la disponibilité en rendant les groupes indépèndants et en leur affectant une certaine autonomie,

- amélioration de la sûreté de fonctionnement par diminution considérable des débits d'information dans le champ et par l'utilisation d'une procédure effectuant des contrôles systématiques au moyen de codes détecteurs d'erreurs performants,

- amélioration de l'aide à la maintenance liée à la modularité de la structure et à la mise en œuvre de tests complexes.

\section{Bibliographie}

[1] Carraud, P., Etude sur la synthèse et l'automatisation des concentrateurs solaires du type "héliostats-tour ". Thèse de Docteur-Ingénieur, 13 novembre 1978, Publication LAAS no 1842 .

[2] Achaibou, A., Bourdeau, C., Massieu, J. L., Pons, J. M., Commande d'un champ d'héliostats par coordonnées calculées. Estimation de la précision de pointage. Note Interne LAAS no 79.1.15, mai 1979.

[3] Mercier, C., Les réflecteurs focalisants. Sécurité relative aux flux THEM 77-31. Documents EDF : Fonctionnement de la centrale THEMIS. 